\section{Herpes hepatitis in pregnancy}

The case of pregnancy associated herpes hepatitis described by Fink et $a l^{1}$ has many similarities with a case we presented to the Medical Society for the Study of Venereal Diseases in Zurich 1993, as well as with other documented cases. However, a literature search has shown that there were 17 reported cases and not eight as stated by the authors. These cases remain rare and the clinical features are initially those of a non-specific viral illness, but it must be borne in mind that herpes simplex is a treatable cause of hepatitis in pregnancy. Including our case, five patients have been treated with acyclovir. All of them survived. This compares with a mortality of $37 \%$ for all cases and $60 \%$ for untreated cases. It must also be noted that not all patients present with florid mucocutaneous lesions and the diagnosis must be considered in their absence. Four of the previous reported 17 cases had no mucocutaneous lesions, while in some, lesions may be scanty and initially overlooked. Even when absent, however, multiple sites are culture positive, and we recommend that oropharyngeal and genital swabs for herpes simplex virus (HSV) should be performed in all cases of hepatitis in pregnancy. Blood cultures may be positive and any biopsy material should also be cultured.

Fink et al suggest that dissemination is not necessarily the result of recent primary infection, but a review of the other documented cases indicates that this is more likely. None of the patients had a clear history of recurrent symptomatic herpes, and in those cases in which antibody responses are documented they are suggestive of primary infection. We know of no cases in which dissemination has been documented in the context of recurrence. This case is also notable as being the earliest stage of gestation in which dissemination has occurred. Other cases have occurred after the 25th week of pregnancy with a mean gestation of 31 weeks.

In summary, disseminated HSV in pregnancy seems to occur in the context of primary infection, usually in the third trimester, but now documented as early as 24 weeks. All cases have been associated with hepatitis; clinical findings may be nonspecific and cutaneous lesions absent. Therefore, a high index of suspicion is needed if antiviral treatment is to reduce the high mortality.

I FAIRLEY

JWILSON

Department of Genitourinary Medicine, The General Infirmary at Leeds, Great George Street,
Leeds LS1 3EX

1 Fink CG, Read SJ, Hopkin J, Peto T, Gould $S$, Kurtz JB. Acute herpes hepatitis in pregnancy. $¥$ Clin Pathol 1993;46:968-71.

Drs Fink and Read comment:

We were most interested to read the letter by Fairley and Wilson reporting other accounts of herpes simplex hepatitis in pregnancy. Unfortunately, we do not have these references to review.

We agree that the condition is rare. It is not the first diagnosis that springs to mind for a pregnant patient who presents with signs of a flu-like illness and subsequent evidence of pneumonia. During pregnancy, liver function may be changed for a variety of reasons some of which are physiological and as in our case, the hepatitis may be fulminant, occurring after a diagnosis of community acquired pneumonia.

Oropharyngeal and genital swabs at the acute stages of the pneumonitis and hepatitis may have isolated HSV from these sites, but as shedding of HSV is associated with pneumonia of bacterial origin ${ }^{2}$ and may be seen intermittently in normal pregnancy, ${ }^{3}$ the diagnostic value for these isolations would be limited.

The correspondents suggest that "blood cultures may be positive". In our patient, in retrospect, the finding of herpes antigen staining in maternal monocytes in one placenta, suggests that we may have been able to culture the virus from a buffy coat preparation. The demonstration of viral antigens using immunoassays or by culture would be a better marker of active virus replication than the polymerase chain reaction (PCR), which may have very limited diagnostic value in this instance because it will show HSV DNA in buffy coat preparations in other patient groups without disseminated herpetic disease. ${ }^{4}$

Fairley and Wilson suggest taking biopsy material for culture. But what tissue would be an appropriate or justified biopsy in a pregnant patient with fulminant hepatitis and severely impaired clotting factors? ${ }^{4} \mathrm{We}$ regret that in our case ${ }^{1}$ the ascitic fluid taken during the hepatitis, which was found to contain monocytes, was not made available for viral culture, although this was a relatively late sample taken after the pregnancy was ended. HSV isolated from this particular material would have been a very strong indicator for an earlier diagnosis and would have been evidence to support starting antiviral treatment

We are aware of reports of the successfu use of acyclovir for the treatment of herpes hepatitis in pregnancy, but it should be remembered that acyclovir is not presently licenced for use in pregnancy. Concerns about its use are not so great for those in the late second or third trimesters, and in disseminated disease intravenous acyclovir would be a justifiable choice. Our difficulty ${ }^{1}$ was that we had no indicator of a diagnosis to be able to start early antiviral treatment which may have preserved the pregnancy.

There may be some misunderstanding by Fairley and Wilson of our use of the term "primary" infection for herpes simplex. Evidence of specific antibody findings in consort studies and surveys in pregnancy 5 suggest that an individual may have been exposed to the virus but that there is an absence of clinical evidence. Mucocutaneous herpetic disease may be seen later on as a first clinical episode, but is not, therefore, a primary infection. For this reason we urge a cautious approach rather than implying that all these cases of hepatitis are necessarily primary infection. This matter may be resolved when more data and good quality differential (HSV 1 and 2) antibody studies are available, but we agree that HSV associated hepatitis has not been recorded in those with a definite history of recurrent herpetic disease except in the immunocompromised.

We support the view of Fairley and Wilson that a higher index of suspicion of disseminated herpes infection in pregnant patients presenting with a pyrexia of unknown origin and $a$ pneumonic and hepatitic picture may permit earlier and thus effective antiviral treatment.
$T$ cell lymphoid aggregates in bone marrow in idiopathic hypereosinophilic syndrome

In a recent issue of the New England fournal of Medicine, there is a report by Cogan et al reporting the presence of clonal proliferation of type II helper T cells in a patient with the hypereosinophilic syndrome. ${ }^{1}$ This is clearly an extremely important and exciting observation. It fully supports the hypothesis put forward by Dr Metz and confirms my own comments relating to the requirement to subdivide $T$ cells on a cytokine basis.

DN SLATER Department of Histopathology, Rotherham District General Hospital, Moorgate Road, Rotherham S60 2UD

1 Cogan E, Schandené L, Crusiaux A, Cochaux P, Velu T, Goldman M. Brief report: clonal proliferation of type 2 helper $T$ cells in a man with hypereosinophilic syndrome. New Engl $¥$ Med 1994;330:535-8.

Seasonal variation in numbers of necropsies with pulmonary embolism

I read with some interest the paper by Green and Edwards, ${ }^{1}$ although I am unable to agree with their conclusion. Having said this, however, I applaud the authors' aim of using one of the rich data sets that are hidden in laboratory records.

The problem here is that the result was not obtained by a formal, global test for seasonality, but instead was apparently found after repeated significance testing of the proportions of necropsies with pulmonary embolism in each month or season. As should be well known by now, under such circumstances the $p$ values obtained must be interpreted with caution-for example, by a Bonferroni type of adjustment-to avoid the risk of wrongly rejecting the null hypothesis. ${ }^{2}$

Although the authors do hint in the introduction that increased morbidity due to cold weather might be a causal factor, they did not make use of this in their analysis, yet if the data had been tested on the basis of this prior hypothesis these would have provided a much more powerful and, if significant, convincing test.

Given that the original analysis can be criticised, what should have been done? One possibility is to use a significance test specifically designed to detect seasonality when the location of the peak is unspecified, on the lines of one described by Edwards. ${ }^{3}$ In its original form Edwards' test is unsuitable because it was designed for counts, so that it is applicable to the total number of necropsies per month rather than the proportion with pulmonary embolism. Using a modified version of Edwards' test suitable for binomial proportions gives a $\chi^{2}$ value of 5.75 (df = 2), p = 0.055-that is, it just fails to reach the $5 \%$ level of significance. On the other hand, if we apply Edwards' original test to the total number of necropsies we obtain a $\chi^{2}$ value of $40.34(\mathrm{df}=2), \mathrm{p}=$ 0.0000 , which is very strong evidence of seasonality. The fitted peak for total numbers of necropsies is around February which would be consistent with a cold weather effect.

Variation in the proportion of necropsies with pulmonary embolism is more likely to 


\section{Correspondence}

Pain, blood loss, and death from leaking abdominal aortic aneurysms

Anthony et al postulate that factors other than the volume of blood loss may play an important role in the pathogenesis of the circulatory collapse which accompanies a ruptured aortic aneurysm. ${ }^{1}$ This may well be so; but I would make the following points about their paper:

1 The leakage of blood into the retroperitoneal space is accompanied by a marked acute inflammatory reaction. The mediators of this reaction have widespread systemic effects.

2 The shock which accompanies leaking aneurysms is associated with marked pulmonary congestion, and often by early "shock lung", both of which will potentiate the effect of any loss of circulating blood volume.

3 The control group presumably included many sudden deaths due to heart disease. The fact that there was no difference between the hearts in the study group and the control group may well only be a reflection of the fact that there is a lot of severe heart disease about!

C G B SIMPSON

Consultant Histopathologist,

Ysbyty Cyffredinol Bronglais,

Bronglais General Hospital, Aberystwyth, Dyfed SY23 1ER
1 Anthony PP, Clarke T, Sarsfield P. Pain, blood loss, and death from leaking abdominal aortic aneurysms. I Clin Pathol 1994; 47:272-3.

Professor Anthony comments:

The points made by Dr Simpson are interesting but no documented evidence is offered from the literature to support them. Our study did not reveal a substantial inflammatory component in response to the presence of blood in the retroperitoneal space, pulmonary congestion was acute, and morphologically severe heart disease was not present in all cases: 15 of 25 had coronary artery stenosis of more than $80 \%$. Death, however, was sudden and occurred within minutes to hours of the onset of symptoms. None of the patients survived beyond admission to the Accident and Emergency Department. These circumstances do not indicate a complicated sequence of events building up over a period of time, nor is it true that it is just a case of there being "a lot of severe heart disease about". Patients who survive "ruptured" or "non-ruptured" abdominal aortic aneurysms after surgical repair do not have a reduced life expectancy when compared with the general population.

\section{Book review}

Heterogeneity of Cancer Cells. Ed M D'Incalci, A Mantovani, S Garattini. Serono Symposia Publications from Raven Press Vol 95. (Pp 154; \$113.50.) Raven Press 1993. ISBN $0-88167-907-0$

This short monograph, published in the Serono Symposia series, reports the proceedings of an International Symposium on Heterogeneity of Cancer Cells held in June 1992 in Milan. A relatively large proportion of the authors are from Italy. As the title implies, the topics concern cell biological and immunological laboratory studies, many in relation to pharmacology, although the final contribution discusses heterogeneity of rumours as a clinical management problem. There are presentations on cell adhesion mechanisms, tumour vasculature and stroma, drug resistance, tumour associated leucocytes, antigen expression, tumour progression in melanoma, growth factor receptors in metastasis, and oncogenes, plus a short index. The articles are all fairly short and vary, as usual, from short reviews to reports of single experiments, but each has a comprehensive bibliography, up to 1992 in some cases. Trivially, but unusually, the formatting of references varies between papers. The book is otherwise well produced, and has appeared reasonably soon after the meeting, but it is rather expensive. It should be of interest to workers in experimental tumour pathology and related fields.

C FISHER

\section{A Residential Course in Histopathology}

22-27 fanuary 1995

Oak Hotel, Brighton, UK

This intensive residential course is part of a three year cycle aimed at providing comprehensive preparation for the MRCPath examination in histopathology, but is equally suitable for consultants wishing to keep informed of recent developments. The course is suitable for 25 cognate points for CME. The programme includes microscopy sessions, discussions, and formal presentations. Registrants will receive a box of 100 stained sections to take away. A mock MRCPath examination is included to give feedback on individual progress.

Speakers include Dr M Boyle (Cytopathology); Professor H Fox (Gynaecological pathology); Dr C Fletcher (Soft tissue pathology); Dr A Hanby (Molecular techniques in diagnosis); Dr N Kirkham (Dermatopathology); Dr C Parkinson (Prostatic pathology); Dr G Stamp (Pancreatic pathology); Professor W Whimster (Pulmonary pathology and morphometry); Dr A Wotherspoon (Lymphoma pathology).

The course fee of $£ 490$ includes accommodation and the slide box.

Details from Dr N Kirkham, P.O. Box 334, Histopathology Department, Royal Sussex County Hospital, Brighton BN2 5BG. Tel: 0273 664501. Fax: 0273 600182 .

\section{Notices}

\section{The Section of Pathology \\ Glycation, Glycosylation and Disease \\ Tuesday 4 October 1994 \\ Programme}

Glycated proteins and diabetes $\mathrm{Dr} E$ Lester, North Middlesex Hospital, London.

The carbohydrate-deficient glycoprotein syndromes: new inborn errors. Clinical and laboratory aspects $D r G$ Keir, Department of Biochemistry, Institute of Neurology, London

Carbohydrate-deficient transferrin as a marker of alcohol misuse $\operatorname{Dr} S B$ Rosalki and $\operatorname{Dr} A Y$ Foo, London

Carbohydrate and protein interaction in inflammation and host defence $\mathrm{Dr} T e n$ Feizi, Cancer Research Campaign, London Glycosylation and tumour markers $G$ A Turner, Department of Clinical Biochemistry, University of Newcastle upon Tyne

Aberrant glycosylation in cancer: potential for immunotherapy Dr foy Burchill, Imperial Cancer Research Fund, London

For further information contact: Miss Claire Cheesman, The Royal Society of Medicine, 1 Wimpole Street, London W1M 8AE. Tel: 071290 2900. Fax: 071 2902989.

\section{Dermatopathology Update \\ UMDS St Thomas's Campus, London}

Friday 27 fanuary 1995

For further details and programme please contact:

Dr P $\quad H \quad$ McKee, Department of Histopathology, St Thomas's Hospital, London SE1 7EH. Tel: 0719299292 (ext: 2039). Fax: 0714013661.

\section{Correction}

Fairley I, Wilson J. Herpes hepatitis in pregnancy (letter). $\mathcal{F}$ Clin Pathol 1994;47:478 The references for the comment by Drs Fink and Read on the above letter were not included with the original manuscript on arrival at this office. The full reference list appears below.

1 Fink CG, Read SJ, Hopkin J, Peto T, Gould $S$, Kurtz JB. Acute herpes hepatitis in pregnancy. $\mathcal{F}$ Clin Pathol 1993;46:968-71.

2 McFarlane JHT. Pneumonia. Medicine International 1991;90:3732-9.

3 Prober CG, Arvin AM. Genital herpes and the pregnant woman. In: Current Clinical Topics in Infectious Diseases. Saunders, 1989.

4 Read SJ. HSV sequences in buffy coat preparations found by PCR in a group of patients with post viral syndrome. 1994. (Unpublished observations.)

5 Mertz GJ, Schmidt O, Jourden JL, Guinan ME, Remington ML, Fahnlander A, et al. Frequency of acquisition of first-episod genital infection with HSV from symptomatic and asymptomatic sourc

6 Whitley RJ, Corey L, Arvin A, Lakeman FD, Sumaya CV, Wright PF, et al. Changing presentation of herpes simplex virus infection in neonates. $\mathcal{F}$ Infect Dis 1988;158: 109-16. 